\title{
Difusor polimérico de lâmpada WLEDi do mercado brasileiro
}

\author{
Polymeric diffuser of WLEDi lamp from the Brazilian market
}

Elvo Calixto Burini Junior ${ }^{1}$, Helio Akira Furuya², Emerson Roberto Santos ${ }^{3,4, *}$, Arnaldo Gakiya Kanashiro

\section{RESUMO}

Lâmpadas com tecnologia a estado sólido (diodo emissor de luz - LED) e base (rosca) tipo Edison possuem difusores poliméricos translúcidos para melhor espalhamento e controle da abertura do facho de luz. A eficiência luminosa da lâmpada WLEDi depende da seletividade espectral e transmitância luminosa do difusor. Duas lâmpadas WLEDi de fabricantes distintos e adquiridas no comércio foram polarizadas por 24 horas/dia até apresentarem falhas e tiveram os difusores investigados com transmitância na faixa de comprimento de onda de 200 até 800 nm comparadas com difusores de outros fabricantes. Diferentes comportamentos de absorção pelos difusores foram identificados, e os espectros de transmitância revelaram que há diferenças na qualidade do material polimérico fabricado nos difusores de lâmpadas para o mesmo fabricante e com diferentes potências nominais. Esta diferença identificada pode ser utilizada como justificativa para diferentes eficiências luminosas nominais descritas nas embalagens dos produtos.

Palavras-chave: Policarbonato (PC), Polimetilmetacrilato (PMMA), Compósito polimérico, Difusor, Lâmpada LED.

\section{ABSTRACT}

Lamps with solid-state technology (light-emitting diode - LED) and base (screw) Edison type have translucent polymeric diffusers for better spreading and control of the light beam opening. The luminous efficiency of the WLEDi lamp depends on the spectral selectivity and light transmittance of the diffuser. Two WLEDi lamps from different companies and purchased in the market were polarized by 24 hours/day until they presented failures and had the diffusers investigated in the range of wavelength from $200 \mathrm{ho} 800 \mathrm{~nm}$ compared with diffusers of other lamp manufacturers. Different absorption behaviors by diffusers were identified, and the transmittance spectral revealed that there is a difference in the quality of polymeric material made of lamp diffusers of the same manufacturer and with different nominal powers. This identified difference can be used as justification for different nominal light efficiencies described on the product packaging.

Keywords: Polycarbonate (PC), Polymethylmethacrylate (PMMA), Polymeric compound, Diffuser, LED lamp.

1. Universidade de São Paulo - Instituto de Energia e Ambiente - São Paulo (SP), Brasil.

2. Faculdade de Tecnologia de São Paulo - Microeletrônica - São Paulo (SP), Brasil.

3. Universidade de São Paulo - Escola Politécnica - Engenharia Metalúrgica e de Materiais - São Paulo (SP), Brasil.

4. Laboratório SuperCriativo - São Paulo (SP), Brasil.

Autor correspondente: emmowalker@yahoo.com.br

Recebido: 30 Nov 2019 Aprovado: 22 Jun 2020 


\section{INTRODUÇÃO}

A lâmpada com emissão de luz branca e com tecnologia a estado sólido (do inglês, solid-state lighting - SSL) está substituindo as lâmpadas incandescente e fluorescente de base única (ou compacta) principalmente no setor de iluminação de interiores ${ }^{1,2}$. Esse tipo de lâmpada possui base (rosca) tipo Edison (E-27) sendo denominada lâmpada LED (do inglês, light-emitting diode). O tempo de vida reduzido dessas lâmpadas LED tem sido observado por consumidores, o que motivou este estudo realizado por meio de um experimento sobre o desempenho.

Em experimento realizado com esses tipos de lâmpadas (consideradas neste trabalho como WLEDi - lâmpadas LED de emissão branca) por Ke e colaboradores, a lâmpada LED foi dividida em diferentes partes, como ilustrada na Fig. $1^{3}$. As três partes principais consideradas por esses autores foram: o driver, o difusor e a fonte de luz (módulo com os LEDs). Eles utilizaram, no experimento, dois conjuntos de lâmpadas LEDs energizadas em: ambiente sob as temperaturas de 25 e $85^{\circ} \mathrm{C}$ e período de teste do tempo de vida de 2.000 horas. A degradação luminosa das lâmpadas apresentou resultados entre 3,8 a 4,9\%, para o grupo sob temperatura de $25^{\circ} \mathrm{C}$, e de 10,6 a $12,7 \%$, para o grupo sob temperatura de $85^{\circ} \mathrm{C}$, mostrando, assim, que a temperatura do ambiente influencia no desempenho da lâmpada LED. O módulo de LED foi considerado a parte mais relevante na comparação entre os conjuntos de lâmpadas, tendo sido responsável pela degradação de 70,5\% em média. O difusor foi o segundo fator relevante da degradação, sendo a causa de $21,5 \%$ em média do total. $\mathrm{O}$ driver foi o terceiro fator de degradação, que representou $6,5 \%$ sob a temperatura de $25^{\circ} \mathrm{C}$ e $2,8 \%$ sob a temperatura $85^{\circ} \mathrm{C}$, em média, do valor total.

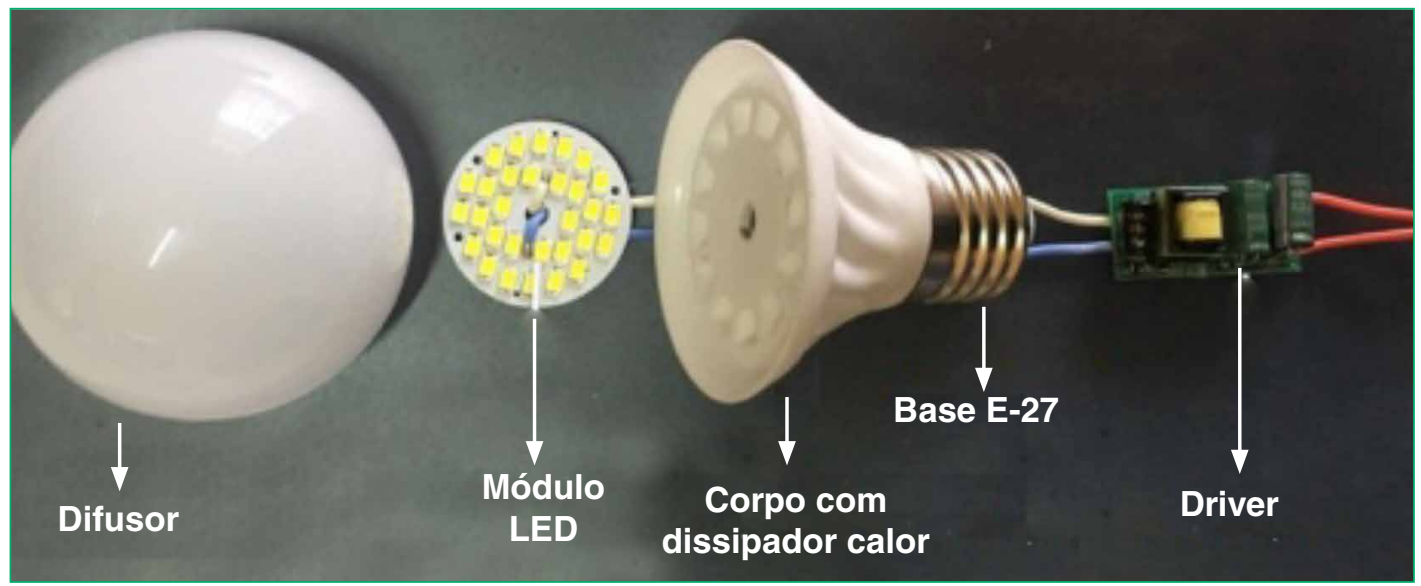

Figura 1: Lâmpada WLEDi com base Edison, subdividida (da esquerda para a direita) em: difusor, módulo LED, corpo (com dissipador de calor e base tipo E-27) e driver (circuito eletrônico) ${ }^{3}$.

Em relação à durabilidade e eficiência luminosa da lâmpada WLEDi, considerando o processo de conversão da energia elétrica em luz visível (energia luminosa), deve-se destacar: o dissipador de calor, elemento que auxilia na transferência do calor do LED, no qual ocorre a conversão energética, e o difusor, que é um material translúcido por onde atravessa a radiação óptica e que realiza a dispersão da luz produzida ${ }^{4,5}$.

A introdução de material fosco utilizado como difusor também possibilita a redução da luminância (intensidade luminosa por unidade de área), e, consequentemente, um possível ofuscamento relacionado à visão do ser humano. Existem difusores de lâmpadas WLEDi fabricados em vidro e em plástico, sendo este segundo utilizado na maioria das lâmpadas comercializadas ${ }^{6,7}$.

Para obterem lâmpadas com maior resistência mecânica ao impacto e menor densidade de material utilizado, os fabricantes de lâmpadas WLEDi têm fabricado difusores com base em materiais poliméricos compósitos. Os compósitos, considerados como estruturas simples, são constituídos por uma fase denominada de matriz (ou base), concebidos de materiais plásticos (polímeros), tais como o polimetilmetacrilato (ou PMMA; densidade de $1,18 \mathrm{~g} / \mathrm{cm}^{3}$ e ponto de fusão de $160^{\circ} \mathrm{C}$ ) e o policarbonato (ou PC; densidade de $1,2 \mathrm{~g} / \mathrm{cm}^{3}$ e ponto de fusão de $140^{\circ} \mathrm{C}$ ), que envolve integralmente o outro elemento/material considerado como a fase dispersa ${ }^{8,9}$.

Os difusores utilizados em lâmpadas WLEDi deverão possuir, principalmente, coeficiente de absorção reduzido e propiciar adequada difusão da luz e, comparando-se o material PC com o PMMA, verifica-se que o PC é superior ao PMMA para a utilização em lâmpadas WLEDi, pelo fato de possuir maior durabilidade e resistência mecânica ao impacto ${ }^{10}$.

A Fig. 2 apresenta o comportamento para a transmissão espectral de luz (ou transmitância) considerado pelo fluxo total da luz para as análises de placas com 03 tipos de materiais diferentes (utilizados como substratos) como: o vidro, o polimetilmetacrilato (PMMA) e o policarbonato (PC), com e sem filme fino aplicado ao substrato e curado. A Fig. 2 foi adaptada do trabalho de Yuan e colaboradores ${ }^{11}$. 


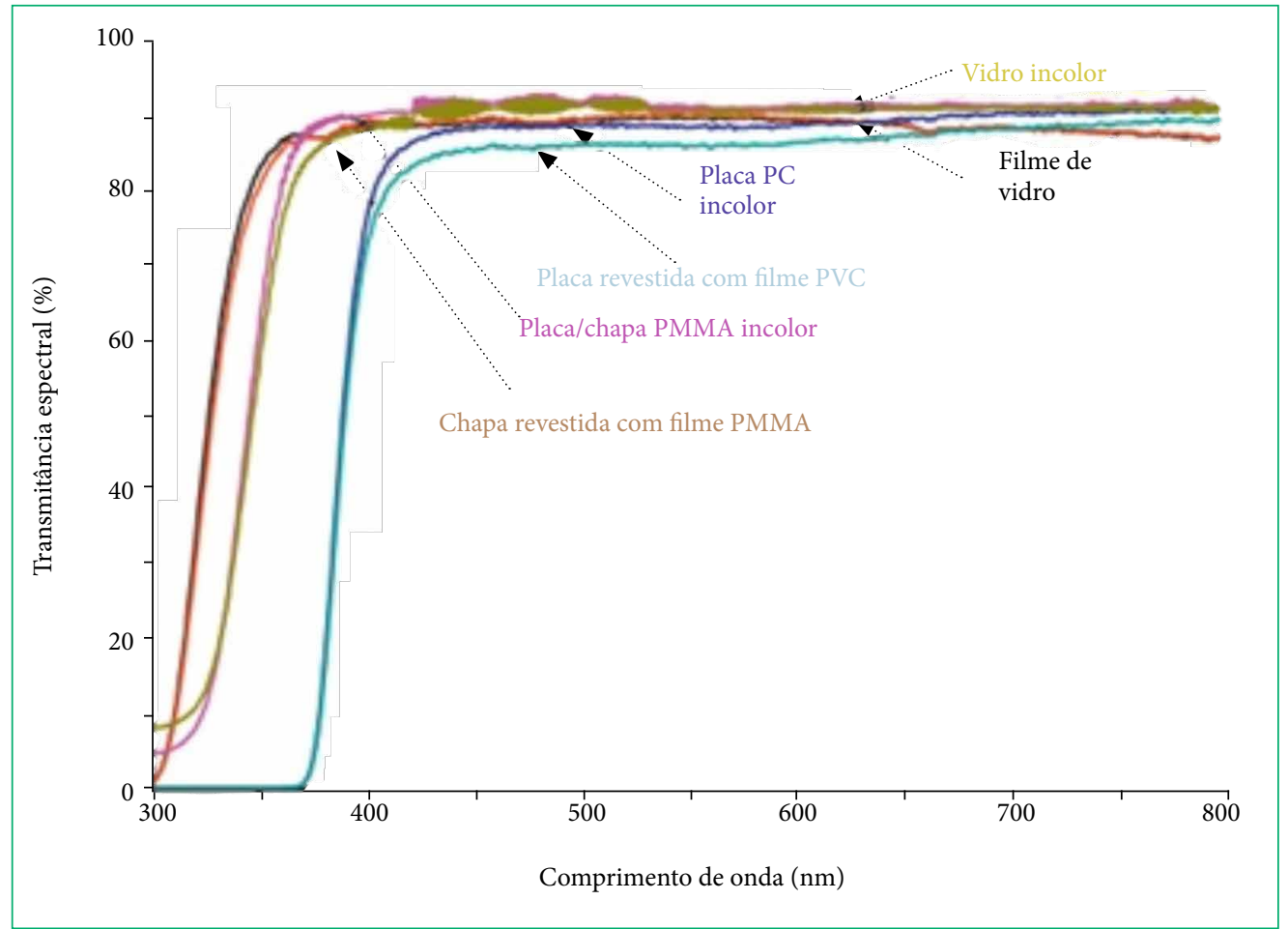

Figura 2: Transmissão espectral de luz através de placas (vidro, PMMA e PC) utilizadas como substrato e tendo (ou não) filme fino depositado e após aplicada cura com UV ${ }^{11}$.

As transmissões ópticas dessas três placas diferentes, utilizadas como substratos, foram investigadas na faixa de comprimento de onda de 300-800 nm. A Fig. 2 (adaptada de Yuan et al. ${ }^{11}$ ) apresenta os espectros nas bandas de radiação: UVA, UVB e visível. Ainda que o autor tenha apontado a existência de desvio reduzido entre a placa original e após o revestimento ter sido aplicado aos três materiais, isso fica mantido enquanto ocorre a redução da "transmitância" espectral. É importante observar os diferentes comportamentos utilizados como "filtros" entre eles. O material PC é aquele que apresenta maior atenuação para a radiação na faixa do UV, o patamar de 50\% de transmissão espectral de luz ocorre a partir de $385 \mathrm{~nm}$ (e apresenta valor de transmitância de $0 \%$, abaixo de $370 \mathrm{~nm}$ ), ele é seguido pelo PMMA (que apresenta $50 \%$ de transmissão a partir de $345 \mathrm{~nm}$, além de apresentar de 5 a $8 \%$ ocorrendo abaixo de $300 \mathrm{~nm}$ ). O vidro comum, ou seja, fabricado em aditivo apresenta o patamar de 50\% de transmissão espectral a partir de $320 \mathrm{~nm}$ (e apresenta valor zero abaixo de $300 \mathrm{~nm}$ ). A variação no caminho óptico, ou seja, a espessura das amostras utilizadas não foi revelada e para comparação entre os resultados foi considerado que as espessuras das placas sejam similares. O fator de transmissão, geralmente, possui comportamento inversamente proporcional à espessura do material. A possibilidade de utilizar o material policarbonato (PC), como difusor de lâmpada WLEDi, é considerada um avanço pelos fabricantes, mesmo que a máxima transmitância teórica de $\sim 86 \%{ }^{12}$, não seja superior nem ao PMMA com $90 \%$ - 92\%, valores típicos ${ }^{12}$, nem ao vidro, que apresenta valor típico de $\sim 90 \%{ }^{12}$.

Segundo Sunderland, o material policarbonato (PC) convencional é material (matriz) que apresenta absorção da luz, o qual após receber aditivo (fase dispersa) passa a apresentar seu desempenho modificado, sendo que o compósito polimérico resultante (como cita o código LED2245 policarbonato) está indicado para ser utilizado como difusor em aplicações de lâmpadas WLEDi ${ }^{13}$.

A fotodegradação é uma das maneiras que pode levar à degradação do material policarbonato (PC), geralmente, pela absorção de radiação na faixa de comprimentos de ondas dos raios ultravioletas (UVs) do espectro eletromagnético ${ }^{14}$. O tipo de material utilizado para compor o compósito polimérico (difusor de luz), fase dispersa, tem sido modificado ao longo do tempo e mantido sob direito de patente registrada.

A literatura indica referências sobre a denominada fase dispersa de o difusor já ter sido constituído de composto inorgânico (como carbonato de cálcio, entre outros) até cerca de 20 anos atrás, quando os compostos orgânicos passaram a ser adicionados basicamente ao material PC usado como matriz ${ }^{15}$. Por exemplo, no caso do PMMA, Xiao e colaboradores formularam uma blenda para ser utilizada como material difusor de luz, a partir do material PMMA dopado com politereftalato de etileno (PET) ${ }^{16}$. Na literatura, existe muito pouca informação sobre a composição e o desempenho referente ao difusor de lâmpadas WLEDi, principalmente sobre lâmpadas do mercado brasileiro. Contudo, a transmissão do difusor desempenha papel relevante, em termos de durabilidade e eficiência luminosa da lâmpada, como confirmado em trabalho anterior, no qual se efetuou experimento de longa duração ${ }^{17}$ com base experimental e com lâmpadas WLEDi novas. 
Assim, neste artigo, o objetivo foi caracterizar as principais propriedades do difusor translúcido de lâmpadas LEDs de emissão de luz branca, determinando as possíveis alterações que o difusor possa apresentar ao longo do período de vida útil, em particular, pelo estabelecimento de metodologia simples capaz de revelar alteração na saída de luz da lâmpada.

\section{MATERIAIS E MÉTODOS}

Em relação às lâmpadas WLEDi com base tipo Edison (rosca E27) analisadas no experimento de longa duração, foram utilizadas: 01 lâmpada da marca Black + Decker e 01 lâmpada da marca Galaxy, ambas com potência nominal de 9 watts. Estas lâmpadas foram adquiridas no comércio varejista e foram energizadas em um suporte metálico contendo porta-lâmpadas durante o período de 24 horas/ dia. Esses experimentos foram realizados sob temperatura ambiente e tensão elétrica estabilizada em $225 \mathrm{~V}$.

Nas análises das transmitâncias dos materiais, foi utilizado um espectrofotômetro da marca Varian, modelo Cary 50 conc UV-Vis. Como materiais de referência, foram analisadas as transmitâncias de 01 placa difusora de material PC semitransparente, obtida de empresa de painéis e displays automotivos; 01 bulbo obtido de uma lâmpada nova (sem utilização) da marca Galaxy de 9 watts; o bulbo de uma lâmpada denominada como Coreana 2 (cujo nome faz referência ao país de origem); o bulbo extraído de 01 lâmpada nomeada como L0, sem marca de origem; além dos bulbos extraídos das lâmpadas WLEDi utilizadas no teste de longa duração.

\section{RESULTADOS E DISCUSSÃO}

Quanto à principal função desempenhada pelo difusor, ele permite aumentar a área de difusão de luz gerada pelo módulo de LEDs, como mostra o exemplo da Fig. 3 para uma lâmpada LED sem o difusor. Quanto à composição, foram encontrados vários registros de patentes para a composição do difusor com base em material PC, entre eles estão, nos EUA: Ohtsuka e colaboradores (1994); Kawato e colaboradores (2009); Nukui e colaboradores (2009); Kawato e colaboradores (2010). Isto indica a importância do conhecimento sobre os diferentes tipos de difusores e seu comportamento óptico, com análises de transmitâncias em diferentes comprimentos de ondas. Portanto, para conhecer melhor sobre a transmitância de materiais difusores e ter-se uma amostra de referência, na Fig. 4 foi obtida a transmitância vs. comprimento de onda para uma placa fina identificada por um fornecedor (empresa montadora de painéis/displays para veículos automotores), sendo definida pelo próprio fabricante como material PC cristal (semitransparente).

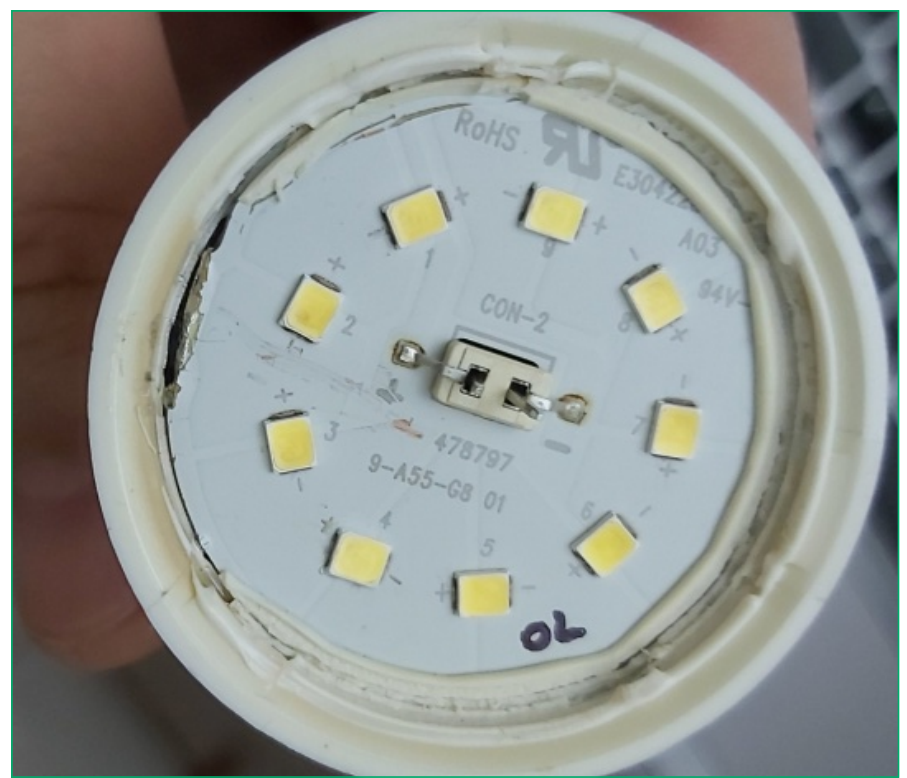

Figura 3:A área de emissão com módulo contendo 9 LEDs de uma lâmpada WLEDi sem o difusor.

O comportamento da transmitância para esta placa fina (Fig. 4) revela valor de 10\% em relação ao máximo ( $86 \%)$ a partir de $400 \mathrm{~nm}$, $50 \%$ de transmissão espectral de luz a partir de $\sim 380 \mathrm{~nm}$ e $0 \%$ abaixo de $\sim 290 \mathrm{~nm}$. Comparando estes três comportamentos em relação aos resultados apresentados na Fig. 2 (resultado retirado da literatura), essa placa fina possui maior semelhança ao material PC, devido à ocorrência de atenuação significativa a partir da banda do UV-A (de 320 a $400 \mathrm{~nm}$ ). 
A Fig. 5 mostra a parte interna do difusor removido da lâmpada WLEDi da marca Galaxy de 9 watts, após 6.339 horas de funcionamento, quando ela apresentou falha catastrófica (inoperante/sem funcionamento e utilizada no teste de longa duração).

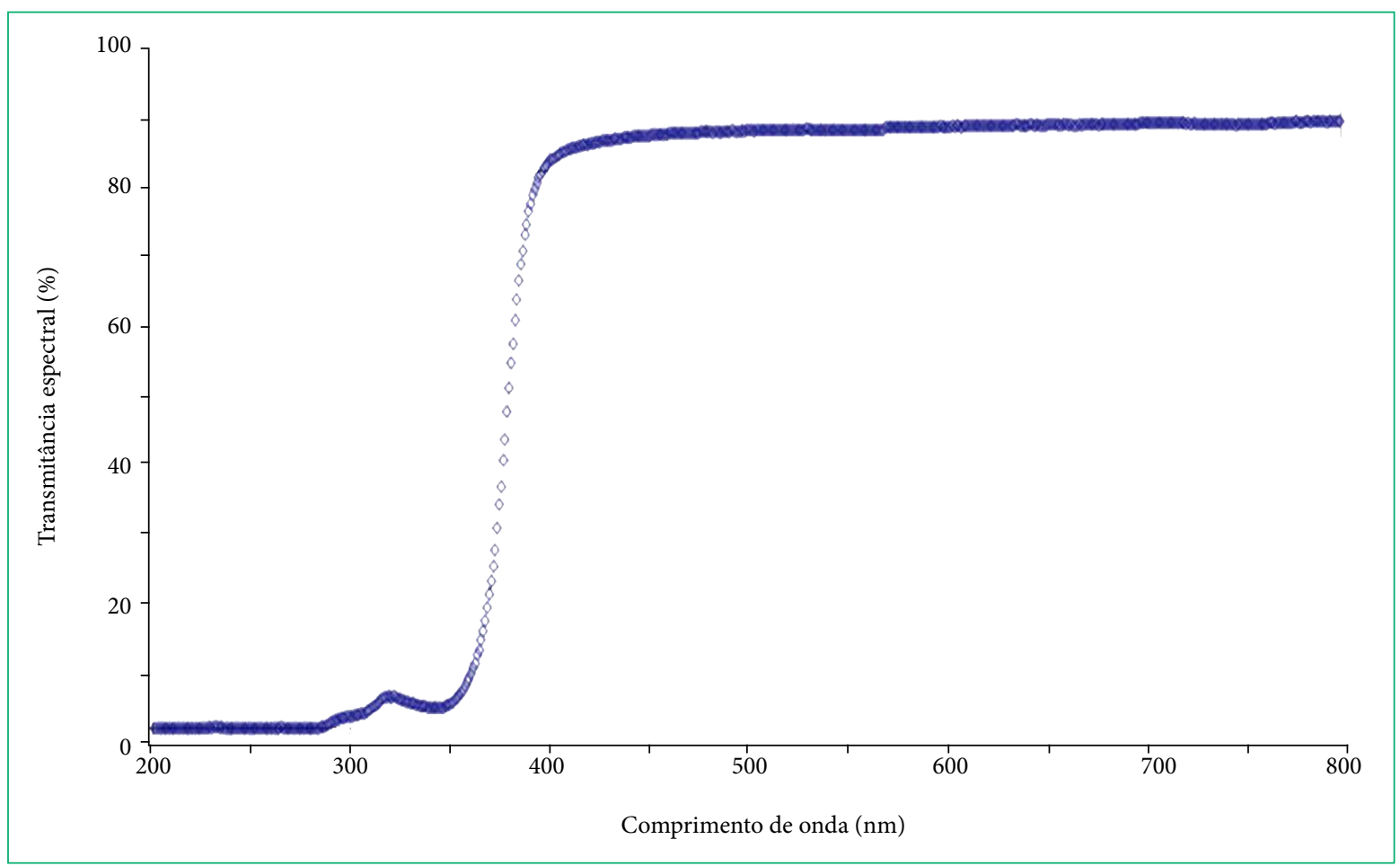

Figura 4: Transmitância vs. comprimento de onda de placa fina identificada como PC cristal (semitransparente).

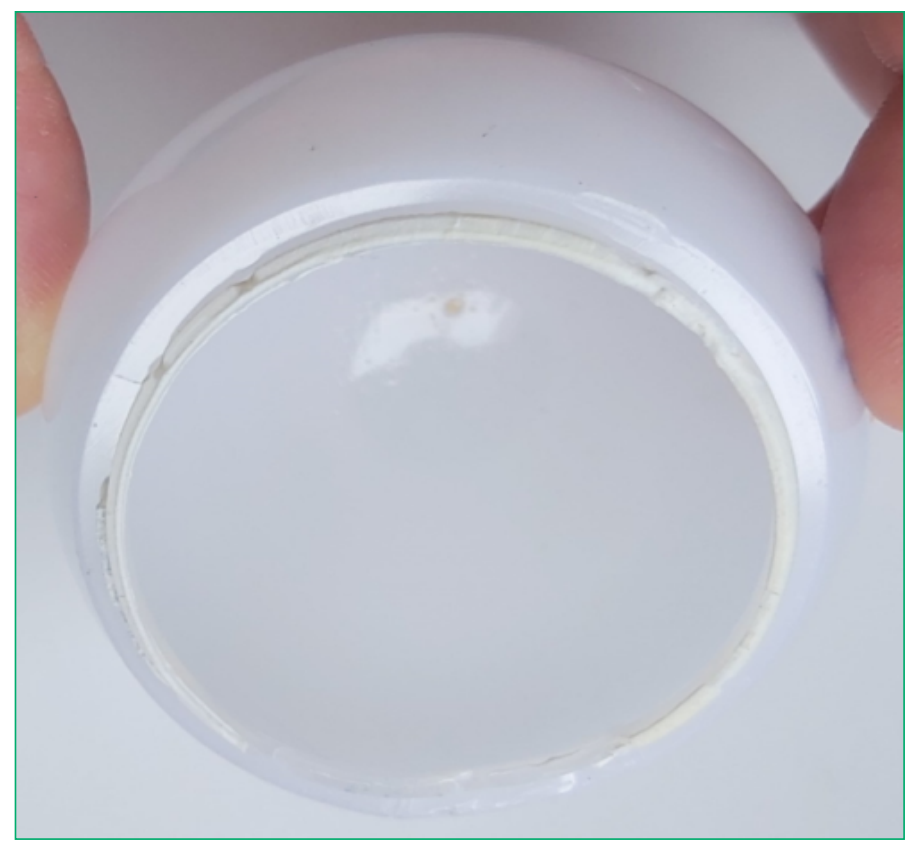

Figura 5: Difusor após ser removido da lâmpada da marca Galaxy de 9 watts com 6.339 horas de funcionamento indicando a "sujeira" oleosa.

No interior do difusor extraído da lâmpada de marca Galaxy de 9 watts (identificado na Fig. 5) foi observada uma substância aparentemente de cor amarelada, cuja hipótese de origem seja do único capacitor eletrolítico (que apresentou estufamento) situado no circuito eletrônico (driver). A transmitância vs. comprimento de onda desse difusor removido foi obtida nessa condição, após ser 
utilizado no teste de longa duração, ou seja, "sujo", com a substância oleosa e também após a tentativa de remoção dessa sujeira, ou seja, "limpo", após a sujeira ser removida com papel absorvente. Também foi medida a transmitância utilizando o difusor de outra lâmpada com as mesmas características elétricas e ópticas do fabricante, ou seja, considerada nova (sem utilização anterior), onde os resultados são apresentados na Fig. 7.

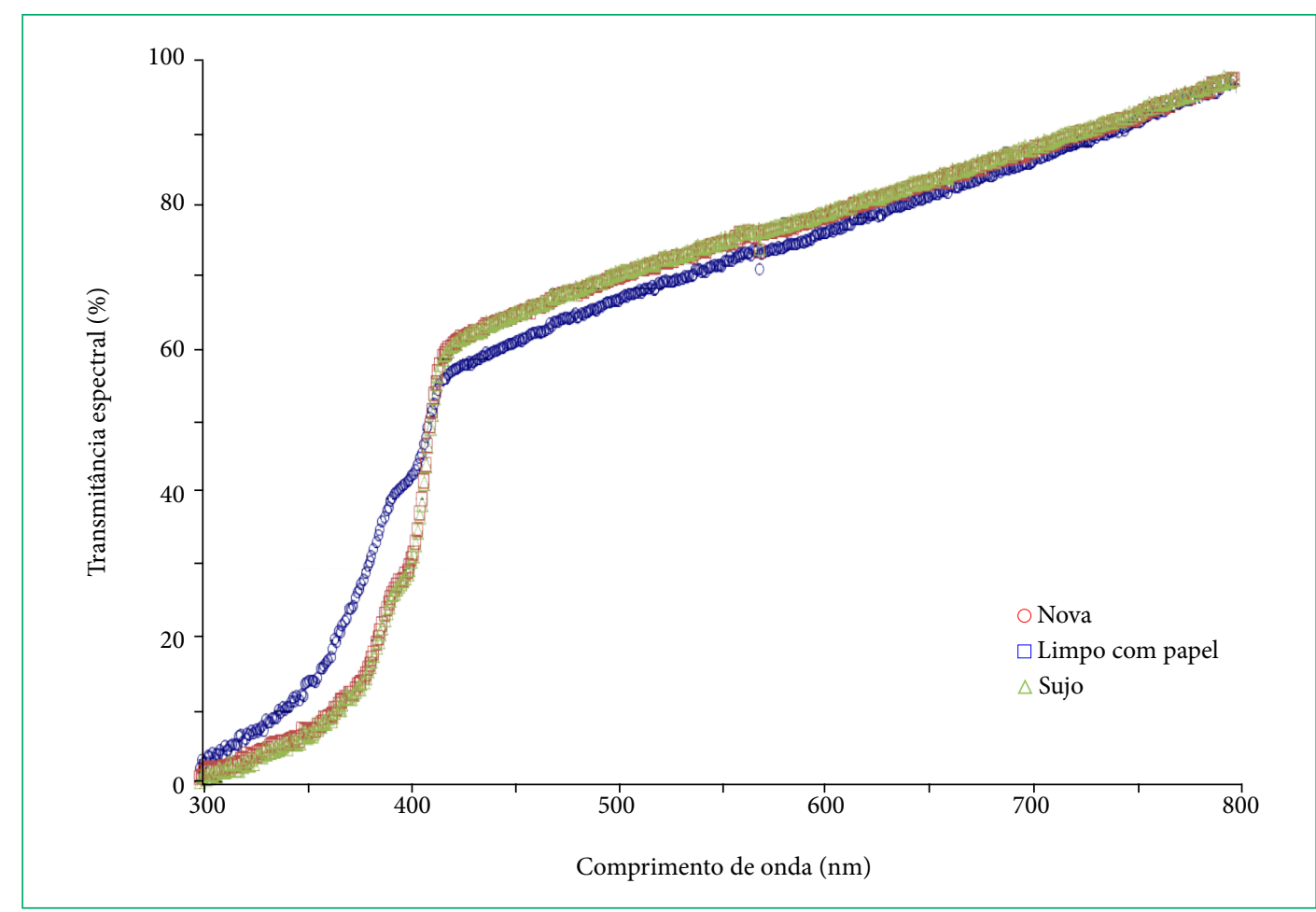

Figura 6: Transmitância vs. comprimento de onda de: difusor removido de lâmpada WLEDi da marca Galaxy de 9 watts nova e WLEDi da marca Galaxy de 9 watts "suja e "limpa", após 6.339 horas de funcionamento até apresentar falha catastrófica (utilizada no teste de longa duração).

A Fig. 6 não revelou diferença significativa na faixa de 300 a $800 \mathrm{~nm}$ da transmitância analisada comparada para o mesmo difusor "sujo" e "limpo". Comparando ambos os resultados de difusores de mesmo fabricante e potência de lâmpada, uma que foi utilizada no teste de longa duração e a outra extraída de uma lâmpada nova, é possível verificar um deslocamento da curva de transmitância para a direita. A substância amarelada que foi encontrada no interior do bulbo da lâmpada "suja" (ou inoperante após 6.339 horas de funcionamento, no teste de longa duração), revelou alteração na transparência do difusor, criando uma espécie de "filtro" para os comprimentos de onda entre 300 e $410 \mathrm{~nm}$. Análises das transmitâncias obtidas para o difusor removido da lâmpada da marca Galaxy de 9 watts que apresentou falha catastrófica (utilizada no experimento de longa duração) e também de outras 03 lâmpadas WLEDi: Black + Decker (comparada no teste de longa duração); Coreana 2 e $\mathrm{L}_{0}$, estão exibidas na Fig. 7.

$\mathrm{Na}$ Fig. 7, a lâmpada identificada como Black + Decker foi submetida ao teste de longa duração e apresentou falha catastrófica após 5.521 horas. Além disso, ela revelou comportamento espectral com base em material PC (espectro similar ao obtido na Figura 4), o material da fase matriz. A lâmpada identificada por Coreana 2 (a qual foi adquirida no ano 2014 e recebeu o nome conforme o país de origem) foi utilizada em residência (até apresentar falha catastrófica), obtendo comportamento totalmente diferenciado nos valores de transmitância do seu bulbo, apresentando variação aproximadamente linear e diretamente proporcional em relação ao aumento com o comprimento de onda (cor lilás no gráfico da Fig. 8). No bulbo da lâmpada Coreana 2, a transmitância começa a aumentar a partir de $300 \mathrm{~nm}$, o que sugere que este material tenha PMMA como matriz. Para auxiliar na análise dos resultados de transmitância apresentados pela Fig. 7, considerando apenas a faixa dos raios UV (mostrado entre 200 a $400 \mathrm{~nm}$ ), representados na Fig. 8, é possível observar o diferencial como maior transmitância pela amostra com identificação $\mathrm{L}_{0}$ (sem marca de origem) e que a Black + Decker seja a única que deve possuir material PC na matriz, apresentando menor transmitância aos comprimentos de onda referente à faixa dos raios UV. Então, os difusores das demais lâmpadas (Galaxy, $\mathrm{L}_{0}$ e Coreana 2) podem ser considerados com material PMMA na matriz. 


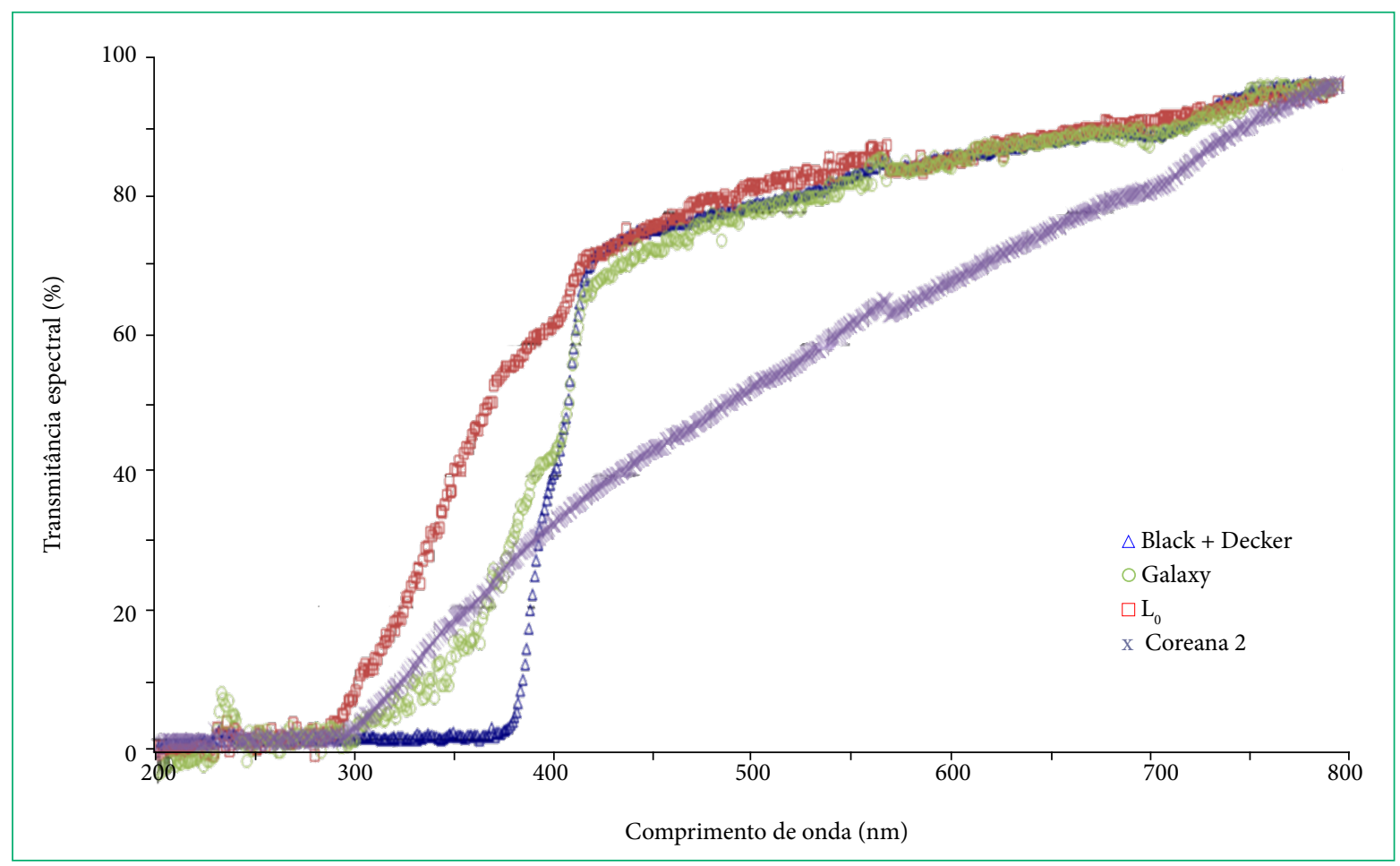

Figura 7: Transmitância vs. comprimento de onda para 04 bulbos de lâmpadas de marcas diferentes.

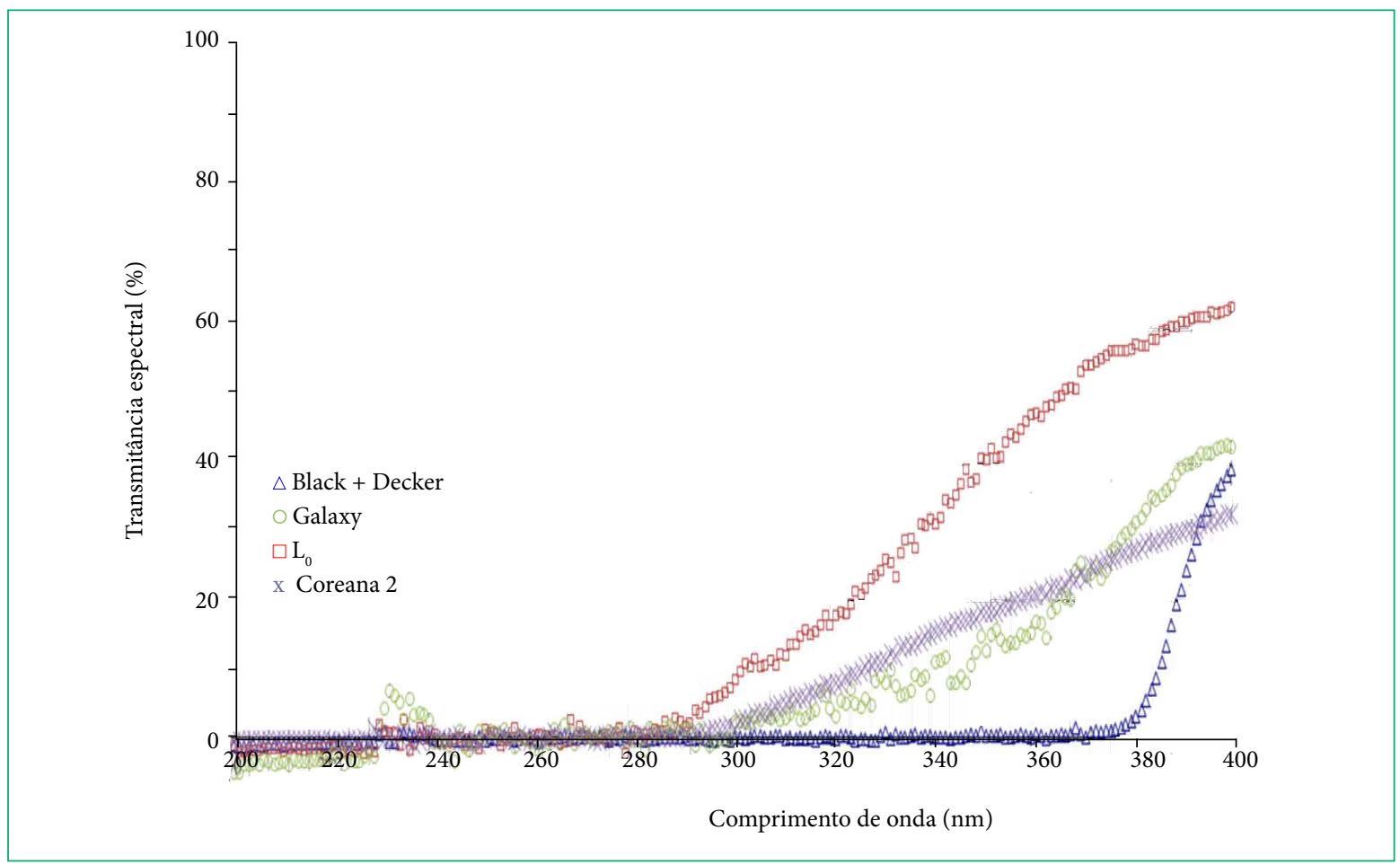

Figura 8: Resultados de transmissão vs. comprimento de onda analisados na faixa de UV para os diferentes difusores.

\section{CONCLUSÃO}

Os registros de patentes sobre a composição do difusor com base em material PC, particularmente nos EUA (obtidos no período entre 1994 e 2010), enfatizam a importância em obter conhecimento sobre os diferentes tipos de difusores utilizados em lâmpadas e o seu 
comportamento óptico, ou seja, em termos da atenuação da luz produzida pelos LEDs nas lâmpadas (WLEDi). Neste trabalho, ainda que não tenha sido constatada a utilização extensiva do material PC, como material da fase matriz (compósitos), utilizado com a função de difusor, o comportamento propiciado pelo material é efetivo como a função de um "filtro" em relação à radiação UV. Contudo, o material mais frequente na fase matriz das amostras foi o PMMA.

Foi verificado que a metodologia utilizada, com medida da transmitância vs. comprimento de onda, possuiu sensibilidade suficiente para identificar alterações entre um difusor novo e outro utilizado sob radiação em lâmpada WLEDi por período superior a 6.300 horas. Porém, não permitiu classificar, com exatidão, o tipo do material utilizado em cada lâmpada WLEDi analisada. A identificação de diferença entre compósitos, matriz em material PC ou material PMMA, é possível a partir das análises de transmitância, tendo como referência em $300 \mathrm{~nm}$ (para o material PMMA) e $370 \mathrm{~nm}$ (para o material PC) para início da transmissão da radiação. As análises de transmitância também revelaram diferentes atenuações nos resultados obtidos entre difusores de uma mesma marca de lâmpada, porém, de potências nominais distintas e também entre diferentes marcas. O resultado observado de maior transmitância foi superior a $10 \%$, porém, decrescente com o aumento do comprimento de onda, o que pode ser utilizado para justificar a eficiência luminosa nominal. Ainda que durante as análises das transmitâncias tenham sido ajustadas iniciando em 100\% para o comprimento de onda em $800 \mathrm{~nm}$, a comparação revela ser materiais diferentes dos difusores analisados, o que sugere ser material policarbonato (PC) o difusor com identificação de 9 watts da lâmpada da marca Black+ Decker e em polimetilmetacrilato (PMMA) os difusores das lâmpadas da marca Galaxy.

Referente ao teste de longa duração foi verificado maior tempo de vida para a lâmpada da marca Galaxy alcançando 6.339 horas em comparação à lâmpada da marca Black + Decker que alcançou 5.521 horas, sendo que ambas atingiram tempos de vida muito menores em comparação às 25.000 horas, como está discriminado nas embalagens dessas lâmpadas WLEDi.

\section{AGRADECIMENTOS}

Este trabalho recebeu a colaboração do Laboratório de Máquinas Elétricas do IEE/USP; da CAPES (Coordenação de Aperfeiçoamento de Pessoal de Nível Superior)pelo fornecimento da bolsa de pós-doutoramento à Engenharia Metalúrgica e de Materiais da Escola Politécnica da Universidade de São Paulo(PMT/USP); e pela infraestrutura e equipamentos utilizados no Laboratório de Engenharia de Macromoléculas.

\section{REFERÊNCIAS}

1. Santos TS, Batista MC, Pozza SA, Rossi LS. Análise da eficiência energética, ambiental e econômica entre lâmpadas de LED e convencionais. Engenharia Sanitária e Ambiental 2015;4(20):595-602. https://doi.org/10.1590/S1413-41522015020040125106

2. Kumar A, Kuppusamy KV, Holuszko M, Song S, Loschiavoc A. LED lamps waste in Canada: Generation and characterization. Resources, Conservation and Recycling 2019;146:329-36. https://doi.org/10.1016/j.resconrec.2019.04.006

3. Ke H-L, Hao J, Tu Jian-Hui, Miao P-X, Wang C-Q, Cui J-Z, Sun Q, Sun R-T. Lumen degradation analysis of LED lamps based on the subsystem isolation method. Applied Optics 2018;57(4):849-54. https://doi.org/10.1364/AO.57.000849

4. Guimarães IAB. Análise e dimensionamento de sistema de iluminação artificial com LEDs para suplementação luminosa no cultivo de Humulus lupulus [trabalho de conclusão de curso na internet]. Juiz de Fora: Universidade federal de Juiz de Fora; 2017. Disponível em https://www.ufjf.br/engenhariaeletrica/files/2018/01/TTC-Inah-Guimarães-Inah-Almeida.pdf

5. Costa VAF, Lopes AMG. Improved radial heat sink for led lamp cooling. Applied Thermal Engineering 2014;70(1)131-38. https://doi. org/10.1016/j.applthermaleng.2014.04.068

6. Leung M, Cree, Inc. White LED and remote phosphor comparison. Cree, Inc. [internet] 2014. Disponível em https://www.cree.com/ledcomponents/media/documents/Remote-Phosphor.pdf

7. Philips Lighting. LED Professional Solutions: Catálogo 2017

8. Serafim BM. Microestruturas porosas de poli(metil metacrilato) depositadas/funcionalizadas por eletrofiação [dissertação na internet] Curitiba: Universidade federal do Paraná; 2016. Disponível em https://acervodigital.ufpr.br/bitstream/handle/1884/43518/R\%20-\%20D\%20 -\%20BRUNO\%20MORAIS\%20SERAFIM. pdf?sequence=1\&isAllowed=y

9. Melo NS. Comportamento mecânico do policarbonato exposto à radiação gama [dissertação na internet]. Rio de Janeiro: Instituto Militar de Engenharia; 2004. Disponível em http://www.ime.eb.mil.br/arquivos/teses/se4/cm/naylor_dissertmest.pdf

10. Sardeiro PS. Parâmetros para a escolha de superfícies translúcidas visando o conforto térmico e visual na edificação [tese na internet] Campinas: Universidade de Campinas; 2007. Disponível em http://repositorio.unicamp.br/bitstream/REPOSIP/258674/1/Sardeiro_ PaulaSilva_D.pdf 
11. Yuan Y, Liu R, Wang C, Luo J, Liu X, Yuan Y. Synthesis of UV-curable acrylate polymer containing sulfonic groups for anti-fog coatings. Progress in Organic Coatings 2014;77(4)785-89. Disponível em http://10.1016/j.porgcoat.2014.01.001

12. Müge Ö. Assessment of lighting performance of PVC and PMMA materials in office spaces in terms of visual comfort [dissertação na internet].[s.I.]: Izmir Institute of Technology; 2006. Disponível em https://openaccess.iyte.edu.tr/bitstream/handle/11147/3703/T000580. pdf?sequence $=1 \&$ is Allowed $=\mathrm{y}$

13. Sunderland N. Shaping LED diffuser performance with polycarbonate materials. 2017. Disponível em <Diffusion 2017 Sunderland Whitepaper_FINAL 2017-05-05.docx>

14. Collin S, Bussière P-O, Thérias S, Lambert J-M, Perdereau J, Gardette J-L. Physicochemical and mechanical impacts of photo-ageing on bisphenolapolycarbonate.PolymerDegradationandStability.2012;97(11):2284-2293. https://doi.org/10.1016/j.polymdegradstab.2012.07.036

15. Smith GB, Jonsson JC, Franklin J. Spectral and global diffuse properties of high-performance translucent polymer sheets for energy efficient lighting and skylights. Applied Optics.2003;42(19)3981-3991. https://doi.org/10.1364/AO.42.003981

16. Liu X, Zhao Z, Xiong Y, Yi P, Guo S. Fast fabrication of a novel transparent PMMA light scattering materials with high haze by doping with ordinary polymer. Applied Optics. 2018;57(9)2107-2114. https://doi.org/10.1364/AO.57.002107

17. Burini Junior EC, Santos ER, Kanashiro AG. Lighting, quality and artificial intelligence [Internet]. Proceedings. 29th CIE SESSION. 2019; Disponível em: http://files.cie.co.at/x046_2019/x046-PO168.pdf 